\title{
Benign Mediastinal Hemangiopericytoma
}

National Cancer Institute

\section{Source}

National Cancer Institute. Benign Mediastinal Hemangiopericytoma. NCI Thesaurus.

Code C6597.

A benign hemangiopericytoma arising from the mediastinum. 